\title{
Probability distribution of drawdowns in risky investments.
}

\author{
Sergei Maslov ${ }^{(1,2)}$ and Yi-Cheng Zhang ${ }^{(2)}$ \\ ${ }^{1}$ Department of Physics, Brookhaven National Laboratory, Upton, New York 11973, USA \\ ${ }^{2}$ Institut de Physique Théorique, Université de Fribourg Pérolles, Fribourg CH-1700, Switzerland
}

(February 1, 2008)

We study the risk criterion for investments based on the drawdown from the maximal value of the capital in the past. Depending on investor's risk attitude, thus his risk exposure, we find that the distribution of these drawdowns follows a general power law. In particular, if the risk exposure is Kellyoptimal, the exponent of this power law has the borderline value of 2 , i.e. the average drawdown is just about to diverge.

For repeated investments one may consider maintaining a fixed fraction $f$ of one's capital in risky assets, while keeping the rest of the capital in risk-free securities. After the pioneering work by Kelly [迎] it is generally believed that the optimal strategy is to choose the investment fraction $f^{*}$ which maximizes the average growth rate of the logarithm of the capital [2]. However, many economists and prudent investors would balk at this aggressive proposal, since this strategy proposes a unique recipe for all purposes, without considering the investor's risk profile. Traditional alternative to the Kelly investment recipe is to select the investment fraction, maximizing the expectation value of some investor-specific utility function [5. Unfortunately, this recipe leads to incorrect expectations, since for very broad distributions, such as log-normal distributions in multiplicative stochastic processes, the expectation value is dominated by an exponentially small fraction of outcomes, and is unlikely to be achieved after a reasonably large number of trials. In the past this common-sense observation has caused persistent debates and was often leading to fallacious conclusions [6].

If the overall shape of utility function is of little relevance in determining the optimal long-term investment strategy, what investment property one should consider to distinguish between aggressive and conservative investment strategies? In this work we systematically study a risk criterion based on the probability distribution of drawdowns of the capital measured relative to its highest value in the past, which we refer to as drawdowns from the maximum. It is often quoted in the trading community that the probability of a given drawdown from the maximum is one of the most sensible parameters of an investment strategy [i]. Often investors identify their wealth as the highest achieved amount. Hence, at any time the current drawdown from the highest capital in the past gives a measure of investor's frustration, tests his strength of nerves and his faith in the ultimate recovery.

The definition of the drawdown from the maximum is rather natural. Let $W(t)$ to denote investor's capital as a function of time. Define $W_{\max }(t)$ to be the overall maximum of the capital up to this point in time: $W_{\max }(t)=$ $\max _{t^{\prime} \leq t} W\left(t^{\prime}\right)$. The current drawdown from the maximum $(\mathrm{DDM}) D(t)$ is given by

$$
D(t)=W_{\max }(t) / W(t) .
$$

From this definition it follows that $D(t) \geq 1$ with equality realized only if the current capital is at its overall maximal value.

Let us first find out the DDM probability distribution in a very general case where the investor's capital follows a discrete-time multiplicative random walk

$$
W(t+1)=e^{\eta(t)} W(t)
$$

In this expression a random number $\eta(t)$ is drawn at each time step $t$ from a given probability distribution $\pi(\eta)$. As usual, it is easier to work with the logarithm of the capital $h(t)=\ln W(t)$, which performs an ordinary random walk

$$
h(t+1)=h(t)+\eta(t) .
$$

The logarithmic drawdown from the maximum (LDDM) $L D(t)=\ln D(t)$ is simply given by

$$
L D(t)=\max _{t^{\prime} \leq t} h\left(t^{\prime}\right)-h(t) .
$$

To the purpose of calculating the probability distribution function of LDDM let us divide the time axis into a sequence of time intervals during which $h_{\max }(t)=\ln W_{\max }(t)$ stays constant (see Fig. 1).

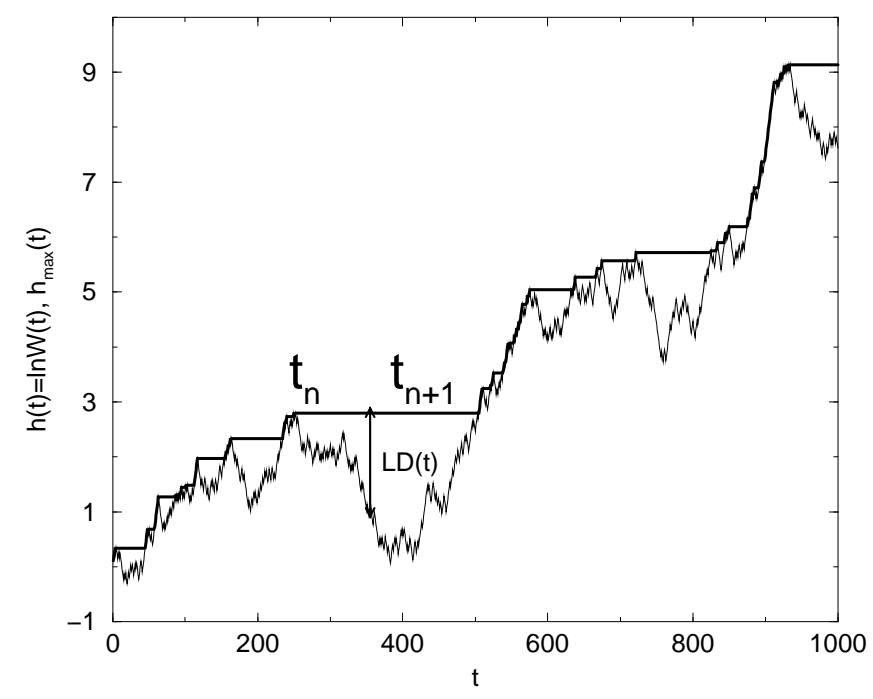

FIG. 1. Random walk $h(t)=\ln W(t)$ (solid line) and its maximal value up to time $t, h_{\max }(t)$ (bold solid line). Between $t_{n}$ and $t_{n+1}$, the maximal value $h_{\max }(t)$ stays constant and is equal to $h\left(t_{n}\right)$. 
Each such interval starts at time $t_{n}$ when the walk is at the overall maximum of $h(t)$ and ends at time $t_{n+1}$ when this maximum is surpassed and replaced by a new, higher one. The motion in each of the intervals $\left(t_{n}, t_{n+1}\right)$ can be viewed as a random walk with an open upper boundary at $h\left(t_{n}\right)$ : the process ends (and the new one starts) when the walk leaves the interval $\left(-\infty, h_{\max }\right)$. Within a single interval without loss of generality we can set $h_{\max }=0$ and, consequently, $L D(t)=-h(t)$. In order to find the distribution of drawdowns from the maximum we need first to calculate the time dependent density $\rho(h, t)$ of the ensemble of random walks in the interval $(-\infty, 0)$ with an open upper boundary. The process starts at $t=0$, when $\rho(h, 0)=\delta(h)$. Note that, since walks can leave the system, the probability of finding a walk within the interval $(-\infty, 0)$ (i.e. of finding the current maximum unsurpassed $t$ time steps after it was realized), $p_{\text {tot }}(t)=\int_{-\infty}^{0} \rho(h, t) d h \leq 1$, is not conserved. Open boundary conditions are equivalent to maintaining $\rho(h, t)=0$ for $h \geq 0$ at all times.

The distribution of drawdowns from the maximum $P(x) d x=\operatorname{Prob}(x<L D<x+d x)$ measures the probability of finding a given logarithmic drawdown at an arbitrary time without any reference to the time $t$ elapsed since the last maximum. Therefore, $P(x)$ is proportional to the time-cumulative density at the point $h_{\max }-x=-x: P(x) \sim \sum_{t=0}^{\infty} \rho(-x, t)$. Including normalization one gets

$$
P(x)=\frac{\sum_{t=0}^{\infty} \rho(-x, t)}{\int_{0}^{\infty} \sum_{t=0}^{\infty} \rho\left(-x^{\prime}, t\right) d x^{\prime}} .
$$

Any ensemble density $\rho(x, t)$ should obey the following integral equation of motion

$$
\rho(x, t)=\int \rho(x-\eta, t-1) \pi(\eta) d \eta
$$

This equation expresses the density $\rho(x, t)$ at time $t$ in terms of the known density at the previous time step $t-1$ and the probability distribution of jumps. It fixes $\rho(x, t)$ for $x \leq 0$, while the definition of an open boundary random walk requires $\rho(x, t)=0$ for $x \geq 0$. The stationary probability distribution of drawdowns from the maximum makes sense only for random walks with a positive (upwards) drift $\langle\eta\rangle>0$. Indeed, for a negative (downwards) drift the maximum realized in the beginning of the process is likely to be never surpassed. In this case as the walk drifts further and further down it samples larger and larger drawdowns so that the probability distribution of drawdowns never becomes stationary. Using the equation of motion (6) for $\rho(h, t)$ one gets $P(x)=A^{-1} \sum_{t=0}^{\infty} \rho(-x, t)=A^{-1}\left(\sum_{t=1}^{\infty} \int \rho(-x-\eta, t-\right.$ 1) $\pi(\eta) d \eta+\rho(-x, 0))=\int P(x+\eta) \pi(\eta) d \eta+A^{-1} \delta(x)$. Here $A=\sum_{t=0}^{\infty} \int_{0}^{\infty} \rho\left(-x^{\prime}, t\right) d x^{\prime}$ is the normalization factor.

Therefore, for $x>0$ one has

$$
P(x)=\int_{-x}^{\infty} P(x+\eta) \pi(\eta) d \eta .
$$

Note also that in order to follow our definition of logarithmic drawdowns we had to change the sign in front of $\eta$, compared to that in Eq. 6. It is a straightforward task to determine the asymptotic behavior of $P(x)$ for $x$ much bigger than the typical value of $\eta$. In this case we can safely disregard that
$P(x)=0$ for $x<0$ and plug the ansatz functional form $P(x) \sim \exp (-\Gamma x)$ into the Eq. 17. The parameter $\Gamma>0$ is then determined from the equation

$$
\int_{-\infty}^{\infty} \pi(\eta) \exp (-\Gamma \eta) d \eta=1 .
$$

One can show that this equation has at most one strictly positive solution. In fact the sufficient and necessary condition for the existence of such solution is a positive upwards drift $\langle\eta\rangle>0$ plus a nonzero support of $\pi(\eta)$ for negative $\eta<0$. Indeed, first one notices that the second derivative of $V(\Gamma)$, where $V(\Gamma)$ is the LHS of Eq. 8 with respect to $\Gamma$, is strictly positive, and $\Gamma=0$ is the obvious solution to $V(\Gamma)=1$. The nonzero $\pi(\eta)$ for some $\eta<0$ guarantees that $V(+\infty)=+\infty$. Since $d V /\left.d \Gamma\right|_{\Gamma=0}=-\langle\eta\rangle<0$, the continuity of $V(\Gamma)$ guarantees the existence of the positive solution of $V(\Gamma)=1$. The positive second derivative ensures its uniqueness.

In order to get a better feeling of how the parameters of the jump distribution $\pi(\eta)$ determine $\Gamma$ we consider two particular functional forms of the distribution $\pi(\eta)$. We first see what happens if $\pi(\eta)$ has a binomial shape. For simplicity let us take a particular binomial distribution, where $\eta=\ln \Lambda$ with probability $p>1 / 2$, and $\eta=-\ln \Lambda$ with probability $1-p$. In other words, with probability $p$ one's capital is multiplied by $\Lambda>1$, otherwise it is divided by $\Lambda$. For this distribution Eq. 8 reduces to $p / y+(1-p) y=1$, where $y=\Lambda^{\Gamma}$. This quadratic equation has two solutions $y_{1}=1$ and $y_{2}=p /(1-p)$. For $p>1 / 2$ (upwards drift condition) the second solution gives the desired positive

$$
\Gamma_{\text {binomial }}=\frac{\ln p-\ln (1-p)}{\ln \Lambda} .
$$

The other case we use to illustrate Eq. 8 is when $\pi(\eta)$ has a Gaussian shape $\pi(\eta)=(1 / \sqrt{2 \pi} \sigma) \exp \left(-(\eta-\mu)^{2} / 2 \sigma^{2}\right)$. Then Eq. 8 can be rewritten as $\exp \left(-\Gamma\left(\mu-\Gamma \sigma^{2} / 2\right)\right)=1$. The unique nontrivial $(\Gamma \neq 0)$ solution, given by

$$
\Gamma_{\text {gaussian }}=\frac{2 \mu}{\sigma^{2}}
$$

is positive, provided $\mu>0$, i.e. the random walk has an upwards drift.

The last equation can be also derived within a continuous time approach. Indeed, increments of a continuous-time random walk, taken at a discrete time intervals, necessarily have a gaussian shape so that Eq. 10 should hold in this case. Another way to see this is to replace the integral equation ( (7) with the differential stationary Fokker-Planck equation

$$
\mu \frac{\partial P(x)}{\partial x}+\frac{\sigma^{2}}{2} \frac{\partial^{2} P(x)}{\partial x^{2}}=0,
$$

where $\mu$, and $\sigma^{2}$ are the drift velocity and the dispersion of the random walk. This equation has a solution $P(x)=$ $A^{-1} \exp (-\Gamma x)$, where $\Gamma$ is given by

$$
\Gamma_{\text {continuous }}=\frac{2 \mu}{\sigma^{2}}
$$

The exponential distribution of logarithmic drawdowns $L D=\ln D$ corresponds to the power law distribution of drawdowns themselves. To properly change variables one notices 
that $\operatorname{Prob}(L D>x) \sim \exp (-\Gamma x)$. Therefore, $\operatorname{Prob}(D>y) \sim$ $y^{-\Gamma}$, and for the distribution of $D$ one has $P(D) \sim 1 / D^{1+\Gamma}$.

It is interesting to note that the mechanism by which drawdowns from the maximum acquire a power law distribution is similar to that of the multiplicative random walk pushed against the wall. This mechanism, which was first analyzed in a financial context by Levy \& Solomon 8 and later studied in greater detail in [9, 10], is rather simple. It is well known that the problem of finding a stationary distribution of a multiplicative random walk drifting in the direction of a reflecting wall can be rewritten in terms of a Fokker-Planck equation for the logarithm of the observable variable with reflecting boundary condition at the position of the wall. The solution of this equation has the well known exponential (Boltzmann) form which, being rewritten in terms of the variable, subject to the multiplicative noise, becomes a power law. In our analytical approach to the problem of drawdowns from the maximum, the current maximal value of the capital serves as an absorbing upper wall for a random walk (once the walk surpasses the current maximum, the value of the maximum has to be updated, which can be looked at as simply taking another representative of the ensemble). Of course, the Fokker-Plank equation with an absorbing boundary, unlike with a reflecting boundary, does not allow for a stationary solution. However, as was demonstrated above, the equations for cumulative (integrated over time) distributions are identical in both cases. That is why it should not be surprising that Eq. 12 of this paper is identical to the Eq. 10 of Ref. [10], which determines the exponent of the stationary power law distribution of multiplicative random walk pushed against the hard wall.

Now we are in a position to derive the distribution of the drawdowns from the maximum for the investor, following a constant investment fraction strategy 1 - 4 . In such a strategy the investor invests a fraction of his capital in one risky asset while keeping the remainder safely in risk-free securities. At each discrete time step the investor sells or buys the correct amount of shares of risky asset to adjust the current value of his asset holdings to precisely the fraction $f$ of his total capital. This investment fraction $f$ (leverage factor if $f>1$ ) is the sole parameter defining the strategy. In this work we do not allow the change of the reinvestment time interval (the discrete time step at which the investor adjusts his asset holdings). Also for simplicity we set the risk-free interest rate to zero. The generalization to a more general situation is rather straightforward, but makes our final formulas less transparent. The evolution of investor's capital for a fixed investment fraction strategy is given by a multiplicative random walk

$$
W(t+1)=W(t)\left(1-f+f e^{\eta(t)}\right) .
$$

In this expression the random variable $\eta(t)$ describes the multiplicative fluctuations of the price $p(t)$ of the risky asset: $p(t+1)=e^{\eta(t)} p(t)$. The results for the distribution of drawdowns from the maximum derived above apply to the fixed investment fraction strategy if one uses $f$-dependent random walk variable $\eta_{f}=\ln \left(1-f+f e^{\eta}\right)$ so that $e^{\eta_{f}}=1-f+f e^{\eta(t)}$. If $f=1$, i.e. the whole capital is invested in risky asset, $\eta_{f}=\eta$ and the whole capital just follows the multiplicative random walk of the risky asset's price. It is clear that by selecting a smaller investment fraction $f$ the investor reduces the probability of significant drawdowns from the maximum, so that $\Gamma_{f}$ is a decreasing function of $f$.
The results are especially straightforward in the case when the logarithm of the stock price follows a continuous gaussian random walk with drift velocity $\mu$ and dispersion $\sigma^{2}$. As it was shown for instance in 邨, for a gaussian $\pi(\eta)$ the logarithm of the capital subject to a fixed investment faction strategy has the drift velocity $\mu_{f}$ and dispersion $\sigma_{f}^{2}$ given by

$$
\begin{aligned}
& \mu_{f}=\left(\mu+\frac{\sigma^{2}}{2}\right) f-\frac{\sigma^{2} f^{2}}{2} ; \\
& \sigma_{f}^{2}=\sigma^{2} f^{2}
\end{aligned}
$$

The exponent of the power law distribution of drawdowns $P(D), \tau_{f}=\Gamma_{f}+1=2 \mu_{f} / \sigma_{f}^{2}+1$, in this case is given by

$$
\tau_{f}^{\text {gaussian }}=\frac{2 \mu+\sigma^{2}}{\sigma^{2} f}
$$

The bigger is this exponent, the safer is your investment from large drawdowns. Of course, the stationary distribution of drawdowns is limited to the case when $\mu_{f}>0$. For the exponent $\tau_{f}$ this corresponds to the condition $\tau_{f}>1$, i.e. normalizable $P(D)$.

It was suggested by Kelly [1] that for the long term investment the optimal fixed investment fraction strategy would be the one maximizing $\mu_{f}$. For the Gaussian $\pi(\eta)$ the investment fraction $f^{*}$ in this Kelly-optimal strategy is given by $f^{*}=1 / 2+\mu / \sigma^{2}$. Indeed, this is what one gets from maximization of the drift velocity $\mu_{f}$ given by Eq. 14. It is interesting to note that for the Kelly optimal strategy the DDM distribution exponent has a superuniversal value

$$
\tau_{f^{*}}=2
$$

This result is not restricted to gaussian $\pi(\eta)$. It is straightforward to demonstrate that it holds at the Kelly optimum for any $\pi(\eta)$. Indeed, by definition of the Kelly optimal strategy it maximizes the growth rate of the logarithm of the capital given by $\mu_{f}=\left\langle\ln \left(1-f+f e^{\eta}\right)\right\rangle$. Therefore, $0=\partial \mu_{f} /\left.\partial f\right|_{f^{*}}=\left\langle\left(e^{\eta}-1\right) /\left(1-f^{*}+f^{*} e^{\eta}\right)\right\rangle=(1-$ $\left.\left\langle\left(1-f^{*}+f^{*} e^{\eta}\right)^{-1}\right\rangle\right) / f^{*}$. From this equation it follows that at the Kelly optimum one has $\left\langle e^{-\eta_{f}}\right\rangle=\left\langle e^{-\ln \left(1-f^{*}+f^{*} e^{\eta}\right)}\right\rangle=$ $\left\langle\left(1-f^{*}+f^{*} e^{\eta}\right)^{-1}\right\rangle=1$, i.e. $\Gamma_{f^{*}}=1\left(\tau_{f^{*}}=\Gamma_{f^{*}}+1=2\right)$ is the solution to (8). That proves that for an arbitrary distribution $\pi(\eta)$ precisely at the Kelly optimum $f^{*}$ the power law distribution of drawdowns has a superuniversal exponent $\tau_{f *}=2$.

Let us illustrate these results using an example of a risky asset, the price of which with equal probability $p=1 / 2$ goes up by $30 \%$ or down by $-24.4 \%$. This is precisely the example of a hypothetical "red chip" stock that we used in "4] to illustrate the power of Kelly optimization. The stock itself is doomed: its price is going down by roughly $1 \%$ every time step (typically at each time step the price is multiplied by $\sqrt{1.30 \cdot 0.756} \approx 0.99)$. On the other hand, since average return of $2.8 \%$ of this stock is positive, following the Kellyoptimal fixed investment fraction strategy with $f^{*} \simeq 0.3825$ results in a positive growth rate of investor's capital of some $0.53 \%$. We have simulated the outcomes of investment process with different investment fractions both above and below Kelly optimal. Fig.2 displays the time dependence of investor's capital for $f=0.1,0.38,0.7$, and 1 . 


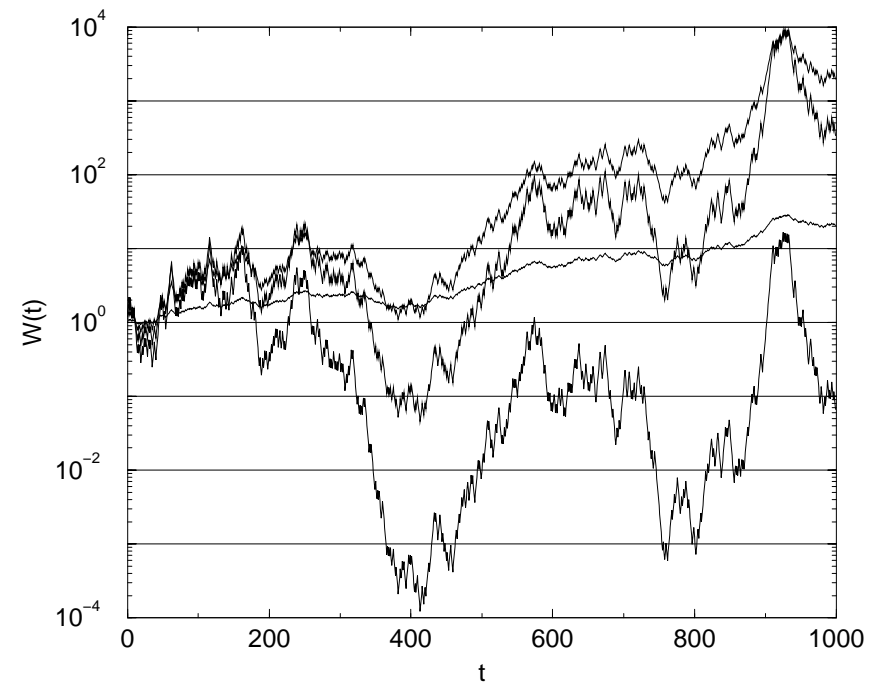

FIG. 2. The evolution of the capital invested in the hypothetical risky asset described in the text at different investment fractions $f=0.38,0.7,0.1,1$ (from top to bottom by the last point $W(1000)$ in the time series) .

It is clear from this figure that the final capital after 1000 time steps grows as $f$ is increased from 0 to 0.38 and starts to go down above 0.38 so that for $f>0.765$ the typical growth rate becomes negative and the investor ends up loosing money. This is illustrated in Fig. 2 on the example of the $f=1$ curve, where the investor trusted his whole capital to the stock and is going down together with this doomed stock.

In Fig. 3 we plot the probability distributions of the drawdowns from the maximum for different investment fractions in this stock.

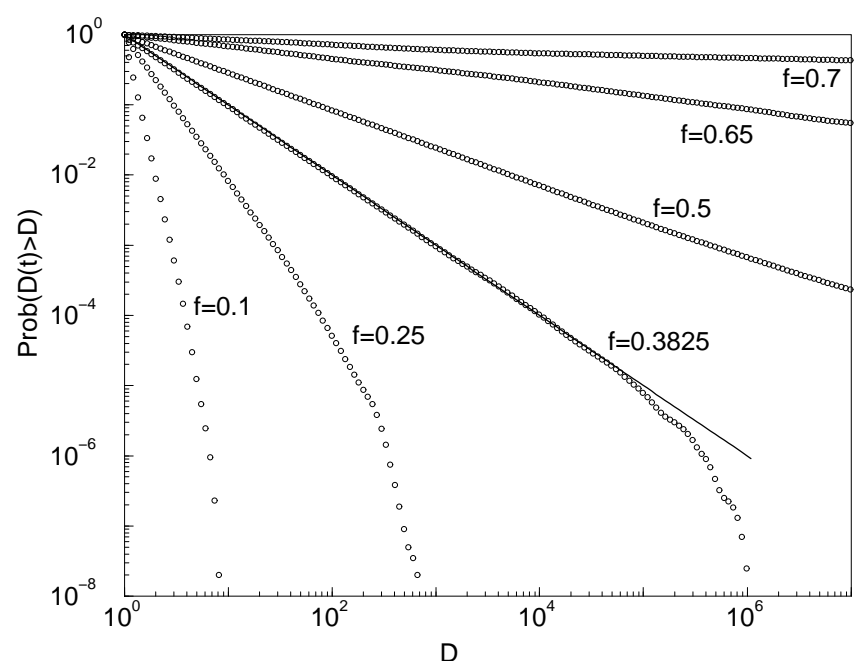

FIG. 3. The probability to have a drawdown bigger than $D$ as a function of $D$ for the same hypothetical risky asset as in Fig. 2. The power law exponent $\tau_{f}$ is systematically decreasing with the investment fraction $f$ ranging from 0.1 to 0.7 . The exponent of the $P(D(t)>D)$ at the Kelly optimum $f^{*}=0.3825$ is in excellent agreement with the theoretical prediction $\Gamma_{f^{*}}=1.5 \times 10^{8}$ data points were used to make histograms in this plot.

The trend of increasing probability of large drawdowns as $f$ is increased can be clearly seen. The $\mathrm{P}(\mathrm{D})$ calculated at the Kelly optimal fraction $f^{*} \simeq 0.3825$ is in agreement with our theoretical prediction of $\Gamma_{f^{*}}=1$.

To illustrate our results on a more concrete example we analyzed the time dependence of the capital invested in S\&P500 index during the year of 1996, using half hourly data provided by Olsen\&Associates. In our hypothetical "investment" we selected and maintained on half-hour basis three different fixed leverage factors: $f=5,10$, and 15 . Any $f>1$, of course, can be realized only if such a leverage ratio is allowed (this is the case e.g. using derivatives such as futures). The resulting drawdown distributions are shown in Fig. 4.

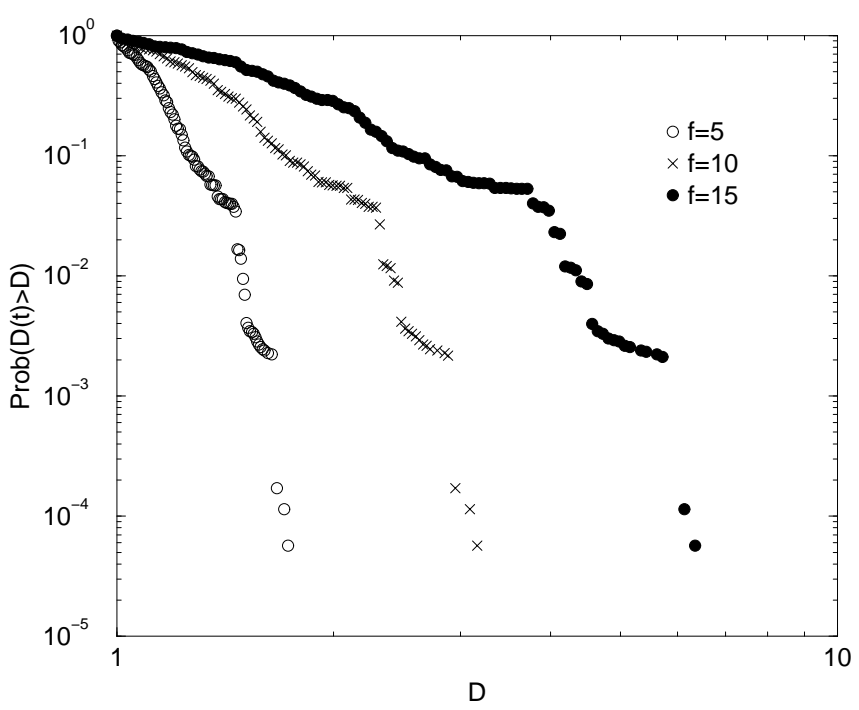

FIG. 4. The probability to have a drawdown bigger than $D$ as a function of $D$ for a leveraged investment in the S\&P500 index during the year of 1996. The investment (leverage) factors are $f=5,10,15$ from left to right.

The largest leverage factor $f=15$ approximately corresponds to the Kelly optimum for this asset under the condition of zero risk-free interest rate.

In summary, we have proposed and studied a risk measure for repeated investment games. We see that, unlike the traditional expected utility approach describing the risk in terms of a single number, we need a whole function to judge if the risk is worth undertaking. Under general conditions this function - the distribution of drawdowns from the maximum - has a power law shape. Kelly's optimal solution represents the most aggressive strategy, since the power law barely gives a finite expectation value of drawdowns (the exponent being 2). More risk-adverse investors can resort to sub-optimal strategies in the Kelly sense, where large drawdowns are considerably tamed. However, even those "safer" strategies are not absolutely free from the risk: since power laws do not have built in cutoffs, ruins (large drawdowns) can in principle arrive but are much less likely.

The work at Brookhaven National Laboratory was supported by the U.S. Department of Energy Division of Material Science, under contract DE-AC02-98CH10886. One of us (S.M.) thanks the Institut de Physique Théorique, Université de Fribourg, for the hospitality and financial support during the visit, when this work was performed. 
[1] J.L. Kelly Jr., The Bell Syst. Techn. Journ. 35 (1956) 917-926.

[2] L. Breiman, Investment Policies for Expanding Businesses Optimal in a Long-Run Sense, Naval Research Logistics Quarterly 7:4 (1960) 647-651; a reprint can be found in Stochastic Optimization Models in Finance, Eds. W.T. Ziemba and R.G. Vickson, Academic Press, New York (1975) p. 593.

[3] E. O. Thorp, a reprint of 1971 Economics Statistics Section Proceedings of the American Statistical Association in Stochastic Optimization Models in Finance, Eds. W.T. Ziemba and R.G. Vickson, Academic Press, New York (1975) p. 599.

[4] S. Maslov, and Y-C. Zhang, Int. J. Theor. and Applied Finance, to appear; cond-mat/9801240.

[5] The optimization of the expectation value of a power law utility function was systematically presented in e.g. R.C. Merton, Continuous Time Finance, Blackwell, Cambridge (1990).

[6] P. A. Samuelson, Proc. Nat. Acad. 68, 2493 (1971).

[7] R. Vince, Mathematics of money management, J Wiley, New York (1992).

[8] M. Levy and S. Solomon, Int. J. Mod. Phys. C7, 595 (1996); S. Solomon and M. Levy, Int. J. Mod. Phys. C7, 745 (1996).

[9] D. Sornette and R. Cont, J. Physique I, 7, 431 (1997).

[10] M. Marsili, S. Maslov, and Y-C. Zhang, Physica A 253, 403 (1998). 